\title{
Continuum Modelling of Granular Particle Flow with Inelastic Inter-Particle Collisions
}

\author{
Yonghao Zhang \\ Department of Computational Science and Engineering, CLRC Daresbury Laboratory, \\ Warrington, UK
}

Jason M. Reese

Department of Mechanical Engineering, King's College London, London, UK

\begin{abstract}
The kinetic theory of granular flow is a successful model for gas-solid flows. However, inelastic collisions between particles, among other mechanisms, cause agglomeration of particles, which may be the reason why undue sensitivity of the model to any slight inelasticity in inter-particle collisions has been seen previously. In contrast to a dry (i.e. no interstitial gas) granular system, this tendency to agglomerate in a gas driven two-phase system may be countered by the carrier gas turbulence. In this paper, a heuristic model for particle gas turbulence interaction is introduced within the scope of a generalised kinetic theory model which incorporates the carrier fluid effect on particulate stresses. The numerical results for the flow of granular particles in vertical pipes, which considers slightly inelastic inter-particle collisions, are in reasonably good agreement with published experimental data. Even in this relatively simple model, the results indicate that the interactions between the particle phase and gas turbulence need to be appropriately addressed in any kinetic theory based model for gas solid flows.
\end{abstract}

Keywords: Particle technology; Gas-solid flow; two-fluid model; turbulence modulation 


\section{Introduction}

In a two-fluid model for gas-solid flows, it is essential task to model the particulate stresses properly. These stresses are generated by direct particle-particle interactions and particle random fluctuational motion. In the last decade, a kinetic theory approach based on "dry" (without gas) granular systems has been widely used to model the particulate phase in gas-solid flows (e.g. Sinclair and Jackson, 1989; Ocone et al. 1993; Nieuwland et al. 1996; Neri and Gidaspow, 2000). However, there are critical differences between a dry granular system and a gas-solid system. In a dry system, the particles tend to coagulate into clusters due to inelastic inter-particle collisions, if liquid bridge and electrostatic forces etc. are negligible. If this agglomeration of particles cannot be dispersed by other mechanisms, a dense clustering will be unavoidable (Goldhirsch and Zanetti, 1993). However, in a gas-solid flow, the interaction between particles and the carrier gas may offset this agglomeration tendency. The equilibrium state of the granular phase therefore depends on the trade-off between inelastic dissipation and particle-gas interactions. Therefore, the correlation between the interstitial gas and the random motion of particles needs to be incorporated into any kinetic model for the particulate stresses. If the effect of interstitial gas can be properly considered, the kinetic theory approach may capture a main generating mechanism of the particulate stresses in a gas-solid system. Consequently, the undue sensitivity to any slight inelasticity in the collisions in dense gas-solid flows, which has been shown in much previous work (Sinclair and Jackson, 1989; Nieuwland et al. 1996; Neri and Gidaspow, 2000), may be avoided. In the present paper, we consider these inter-phase interactions within the scope of a two-fluid model, and the numerical solution of our model for dense gas-solid flows in vertical pipes are compared with published experimental data and other simulation results. 


\section{Mathematical model}

In a two-fluid model, the momentum equations for both phases can be given as (Anderson and Jackson, 1967):

$\underline{\text { solid phase }}$

$$
\varepsilon_{2} \rho_{2} \frac{D}{D t} \mathbf{U}=-\varepsilon_{2} \nabla P-\nabla \cdot \tau_{2}+\boldsymbol{F}_{d r a g}+\varepsilon_{2} \rho_{2} \boldsymbol{g}
$$

gas phase

$$
\varepsilon_{1} \rho_{1} \frac{D}{D t} \boldsymbol{V}=-\varepsilon_{1} \nabla P-\nabla \cdot \tau_{1}-\boldsymbol{F}_{\text {drag }}
$$

where subscripts 1 and 2 represent the gas phase and solid phase respectively, e.g., $\varepsilon_{1}$ and $\varepsilon_{2}$ are the volume fractions of the gas phase and the particle phase; $\rho$ is the density; $\tau$ is the stress; $\boldsymbol{U}$ is the averaged velocity of the solid phase; $\boldsymbol{V}$ is the averaged velocity of the gas phase; $\boldsymbol{F}_{d r a g}$ is the averaged drag force; $P$ is the pressure; $\boldsymbol{g}$ is the gravitational acceleration. In equation (2), the body force on the gas phase is neglected because of its low density.

According to Elghobashi (1994), when the solid volume fraction is greater than $0.1 \%$, the flow may be classified as "dense", and the collisions between particles cannot be ignored. With increasing solid volume fraction the inter-particle collisions, rather than gas turbulence, dominate the flow. Zaichik et al. (1997) proposed a generalized kinetic model which accounted for both inter-particle collisions and particle-gas interactions via a Boltzmann integral operator and a generalized Fokker-Planck differential operator respectively. Peirano and Leckner (1998) derived a competing kinetic theory model of granular flow including a turbulent interstitial gas, based on the work of Jenkins and Richman (1985).

Here, as it is most appropriate, we will adopt the work of Peirano and Leckner to model the particulate stress, i.e. 


$$
\tau_{2 i j}=\left(P_{2}-\zeta_{2} S_{k k}\right) \delta_{i j}-2 \varepsilon_{2} \rho_{2}\left(\psi_{2}^{c}+\psi_{2}^{t}\right) \hat{S}_{i j}
$$

where the particle normal stress is

$$
P_{2}=\varepsilon_{2} \rho_{2}\left(1+2 \varepsilon_{2} \chi(1+e)\right) T
$$

where $S_{2 i j}=\left(U_{i, j}+U_{j, i}\right) / 2, \hat{S}_{2 i j}=S_{2 i j}-S_{2 m m} \delta_{i j} / 3$ and $e$ is the particle-particle collisional coefficient of restitution; $\zeta_{2}, \psi_{2}^{c}$ and $\psi_{2}^{t}$ are the bulk viscosity, the collisional viscosity and the turbulent viscosity respectively, which are given in Appendix $\mathrm{A} ; \chi$ is the radial distribution function; $T$ is granular temperature defined as $T=\left\langle\boldsymbol{u}^{\prime} \cdot \boldsymbol{u}^{\prime}\right\rangle / 3$ and $\boldsymbol{u}^{\prime}$ is the particle fluctuational velocity; $\delta_{i j}$ is the Kronecker delta given by

$$
\delta_{i j}= \begin{cases}0, & i \neq j \\ 1, & i=j .\end{cases}
$$

A closure equation for the balance of the fluctuational energy is needed:

$$
\frac{3}{2} \varepsilon_{2} \rho_{2} \frac{D}{D t} T=-\nabla \cdot \boldsymbol{q}-\tau_{2}: \nabla \boldsymbol{U}-\beta_{0}\left(3 T-\left\langle\boldsymbol{v}^{\prime} \cdot \boldsymbol{u}^{\prime}\right\rangle\right)+I
$$

where $v^{\prime}$ is the fluctuational velocity of gas; $\beta_{0}$ is the effective inter-phase momentum transfer coefficient for drag. The expression for the fluctuational energy flux, $\boldsymbol{q}$, is given by Peirano and Leckner (1998), viz.

$$
\boldsymbol{q}=-\frac{3}{2} \varepsilon_{2} \rho_{2}\left(\kappa_{2}^{t}+\kappa_{2}^{c}\right) \nabla T
$$

The diffusion coefficients, $\kappa_{2}^{c}$ and $\kappa_{2}^{t}$, are given in Appendix A. Energy dissipation due to inelastic collisions is described by

$$
I=12 \varepsilon_{2}^{2}\left(e^{2}-1\right) \chi \rho_{2} \frac{1}{d \sqrt{\pi}} T^{\frac{3}{2}} \text {. }
$$

The radial distribution function $\chi$ is described by the expression of Lun and Savage (1987) viz.

$$
\chi=\left[1-\frac{\varepsilon_{2}}{\varepsilon_{2 m}}\right]^{-2.5 \varepsilon_{2 m}},
$$


where $\varepsilon_{2 \mathrm{~m}}$ represents the maximum possible particle fraction of the system, i.e. 0.645 in the calculation.

The velocity correlation between the gas and particulate phases is essential in determining the balance of fluctuational energy, as well as particulate viscosity and diffusion coefficients. Koch (1990) derived an equation for this energy source in a dilute gas-solid suspension in a Stokes flow:

$$
\beta_{0}\left\langle v^{\prime} \cdot u^{\prime}\right\rangle=\frac{\rho_{s} d \varepsilon_{2}(U-\boldsymbol{V})^{2}}{4 \sqrt{\pi} t_{s}{ }^{2} \sqrt{T}},
$$

where $d$ is the particle diameter; and $t_{s}$ is the Stokes relaxation time. Koch and Sangani (1999) extended the early work of Koch (1990) to dense gas-solid flows, and argued that when the particle Reynolds number is small and the mean particle collision interval is smaller than the particle viscous relaxation time, this energy source is related to the autocorrelation of the force felt by a test particle. They expected this correlation time to be of the order of $0.5 \mathrm{~d} / T^{1 / 2}$, the time over which the spatial configuration of particles changes significantly. The force on a particle was assumed to be of the order of $3 \pi \mu d U$ for a zero slip velocity flow, where $\mu$ is the gas viscosity.

For a gas solid flow with relatively large particle Reynolds number, the force acting on an individual particle is difficult to determine, especially when the driving gas is turbulent. Brucato et al. (1998) stated that the drag coefficient could be significantly affected by the carrier fluid turbulence: compared to the standard drag curve, their experimental data showed the drag coefficient could be 40 times greater in a high turbulent intensity. Therefore, the particle Reynolds number alone may be insufficient to characterise the drag force. When Re is about $10^{5}$, the proportion of the total drag resulting from skin friction is only about $1.3 \%$ (Clift et al. 1978). 
In a dense gas-solid flow, the mean interval between particle collisions is much shorter than the particle turbulent response time, so the particles are therefore actually continuously accelerating. Also, the collisions between particles change the flow state of surrounding gas dramatically. Thus, other forces including added mass and lift force etc. need to be considered as well. Although the drag forces proposed by Ergun (1952) and Wen and Yu (1966) have been commonly applied in modelling gas-solid flows by the two most widely-used methods, i.e. discrete particle simulation and the two-fluid approach, both formulas only addressed the effect of voidage on the drag force. Other factors such as particle random motion and inter-particle collisions, as well as carrier gas turbulence, need to be addressed (Zhang and Reese 2003). As no satisfactory work on the general drag force in a dense gas-solid flow exists at present, we extend the work of Koch (1999) by assuming the force acting on a particle from a turbulent gas at large particle Reynolds number could be comparable to the order of $3 \pi\left(\mu+\mu_{t}\right) d|\boldsymbol{u}-\boldsymbol{v}|$, where $\mu_{t}$ is the gas eddy viscosity. This is a first estimate, which may give the right order-of-magnitude sensitivity for the force acting on the particles from the carrier gas.

Building on this argument, we propose extending equation (9) to a flow with a high particle Reynolds number by using an energy source of the form

$$
\beta_{0}\left\langle\boldsymbol{u}^{\prime} \cdot \boldsymbol{v}^{\prime}\right\rangle=\frac{d\left[3 \pi\left(\mu+\mu_{t}\right) d\right]^{2}<(\boldsymbol{u}-\boldsymbol{v})^{2}>}{\frac{1}{2}^{\frac{1}{2}}},
$$

where $m$ is the mass of a particle. For a homogenous turbulent gas flow, the mean square slip velocity is given by Zhang and Reese (2001),

$$
\left\langle(\boldsymbol{u}-\boldsymbol{v})^{2}\right\rangle \approx(\boldsymbol{U}-\boldsymbol{V})^{2}+\frac{8 T}{\pi} .
$$

From equations (10) and (11), we can see that the energy flux between the two phases has two components: one caused by the mean slip velocity, the other due to the random motion of the 
particles. This approach is in agreement with Koch (1990), who noted an energy source could arise from the random forces acting on a particle due to hydrodynamic disturbances by the neighbouring particles when the mean slip velocity between the two phases is zero.

The gas turbulence will be affected by the random motion of particles due to inter-particle collisions, and the mechanisms of this turbulence modulation are still not clear. Many factors, such as particle size, inertia and volume fraction, may be relevant. Generally, experimental data shows that larger particles tend to enhance the turbulence and smaller particles attenuate it (Gore and Crowe, 1989). Here we consider small particles (although high inertia), so that the turbulence-inducing wakes can be ignored and the gas turbulence will be attenuated. We introduce a correction function, $\psi\left(\varepsilon_{2}\right)$, which modifies the eddy viscosity, reducing it by a factor of $\psi\left(\varepsilon_{2}\right)$ due to the presence of particles, i.e. $\mu_{t}^{*}=\mu_{t} \psi\left(\varepsilon_{2}\right)$. Because of the lack of information on gas turbulence modulation by particles, and the scale difference between the two phases, high order turbulent models such as $k-\varepsilon$ may not be suitable for describing gas turbulence at this stage. However, for a simple pipe flow, a zero-equation model, such as the mixing length model, has been well tested and is likely to be sufficient to capture the main flow characteristics. Therefore, here a mixing length model is adopted to determine the eddy viscosity, $\mu_{t}$.

The mixing length may be interpreted as the distance that a gas molecule cluster could randomly travel in a turbulent flow. However, the presence of particles will restrict the random motion of the gas phase, thereby reducing the mixing length. The correction function, $\psi\left(\varepsilon_{2}\right)$, should reflect this reduction. In a unit volume of a two-phase mixture, the particle phase occupies a volume of $\varepsilon_{2}$. Hence, the presence of the small particles reduces the space available for the random travel of gas molecule clusters, and hence the mixing length, i.e. 


$$
\psi\left(\varepsilon_{2}\right)=\left(1-\varepsilon_{2}{ }^{1 / 3}\right)^{2}
$$

Although this approach is based on physical argument rather than rigorous derivation, it is sufficient as a first approximation to the complex effect of gas turbulence modulation. It has the advantage of simplicity; its utility and accuracy will be tested numerically below. Finally, the averaged drag force is (Zhang and Reese, 2003)

$$
\boldsymbol{F}_{\text {drag }}=\beta_{0}(\boldsymbol{V}-\boldsymbol{U}) .
$$

\section{Numerical results and discussion}

In the simulation, a modified wall function, as given by Louge et al. (1991), is adopted as the boundary condition for gas turbulence, and other boundary conditions for fully-developed granular particle flow in a vertical pipe are as in Zhang and Reese (2001). In the past three decades, many experiments have been carried out to measure solid-gas flow in a vertical pipe or a riser, however, very little data has been published of simultaneous measurements of the radial profiles of solid concentration, local mass flow rate or solid velocity. Recently, Miller and Gidaspow (1992) measured solid volume fraction and mass flux profiles simultaneously in dense particulate flows. The mass flux was measured by means of an extraction probe, and particle concentrations were measured by an X-ray densitometer. Nieuwland et al. (1996b) used a reflective optical fibre probe to measure solid concentration and solid axial velocity profiles. Currently, these two sets of instantaneously measured data are the most comprehensive in the dense gas-solid flow regime. Because the solid particles used in the measurements of Miller and Gidaspow (1992) are FCC with $75 \mu \mathrm{m}$ mean diameter (which are in Group A), the inter-particle contact forces, such as van der Waals force and electrostatic forces may also play a significant role in the flow (Ocone et al. 2000). These inter-particle forces are not accounted for in the present model for the particulate stresses. Therefore, we will compare our results only with the measurements of Nieuwland et al. (1996b), where Group B particles are used and inter-particle 
contact forces are negligible. In these experiments, the mean particle collision interval, $t_{2}^{c}$, is larger than $0.5 \mathrm{~ms}$, the smallest particle relaxation time scale, $t_{12}^{x}$, is about $150 \mathrm{~ms}$, while the slowest large eddy time scale, $t_{1}^{t}$, is of the order $R / \bar{V} \sim 10 \mathrm{~ms}$ (Louge et al. 1991). It is clear that the inter-particle collisions are dominating the flows and the particles do not follow the gas turbulence. Therefore, for dense gas-solid flows with the high inertia particles considered here, the interstitial gas turbulence has negligible effect on the random microscopic motion of particles (although the particles modulate the turbulence). Consequently, the model of Peirano and Leckner is identical to the work of Jenkins and Richman (1985) and Lun et al. (1984). The essential physical properties and model parameters of the system under investigation are collated in Table 1.

In Figure 1, the superficial gas velocity, $V_{s}$, is $14.4 \mathrm{~ms}^{-1}$ and the imposed solid mass flux, $G_{s 0}$, is $350 \mathrm{kgm}^{-2} \mathrm{~s}^{-1}$. Nieuwland et al. (1996a) ignored the kinetic fluctuational energy production due to gas turbulence so that their model failed in prediction for even very slightly inelastic mutual particle collisions, e.g. $e=0.999$. In the present model, we have considered the fluctuational energy dissipated not only by inelastic collisions between particles, but also by viscous friction with the gas phase. Moreover, we have also considered the fluctuational energy generated by interactions with the turbulent gas. (If we ignore the fluctuational energy source, we too fail to predict any solid lateral segregation as Nieuwland et al. (1996a) did even for $e=0.999$.)

Figure 1(a) shows that the results of the present model for the solid volume fraction profile agree well with both the experimental data and simulation results of Nieuwland et al. (1996a, b). Comparisons of solid velocity profiles can be seen in Figure 1(b). The profiles of both numerical results are in reasonably good quantitative agreement with the experimental data. The present model predicts a higher solid velocity in the pipe centre than does the numerical 
solution of Nieuwland et al. (1996a). It should be noted, however, that unphysically elastic inter-particle collisions are assumed in the work of Nieuwland et al. (1996a), while the results of our present model take the collisions to be slightly inelastic.

Figures 2 - 4 show comparisons of the solid volume fraction and particle axial velocity, where the superficial gas velocity is $10 \mathrm{~ms}^{-1}$, and the imposed solid mass flow rates are 400, 300 and $200 \mathrm{kgm}^{-2} \mathrm{~s}^{-1}$ respectively. Again, the simulation results are in good agreement with measured data. This indicates that the kinetic theory model, which has very few empirical parameters, may capture the gas solid flow characteristics despite needing to accommodate an impact velocity-dependent coefficient of restitution. The present model predicts a much smaller slip velocity than the model of Nieuwland et al. (1996a), which leads to a smaller pressure drop. However, the present model still over-predicts the pressure drop significantly. For example, for the operating conditions as given in figure 3, the model estimated the pressure drop as 2000 $\mathrm{Pam}^{-1}$, while the experimental measurement is about $1000 \mathrm{Pam}^{-1}$. This discrepancy could be partly due to the fact that the experimentally-measured mean solid mass flow rate, which depends on the measured solid velocity and the solid volume fraction, is much higher than the imposed solid mass flow rate.

The 'similar profiles' regime was first proposed by Monceaux et al. (1986), and has been more recently reported by other researchers (e.g. Rhodes et al. 1992). It can also be confirmed here. Figure 5 shows that the profiles of the reduced solid flux, $G_{r} / G_{s}$ (which is the local solids flux divided by the mean flow rate), are relatively insensitive to changes of the mean solid mass flow rate at a given superficial gas velocity. The superficial gas velocity is $10 \mathrm{~ms}^{-1}$ and the imposed mass flow rates are 400, 300 and $200 \mathrm{kgm}^{-2} \mathrm{~s}^{-1}$ respectively. The other parameters are given in Table 1. The model prediction of pressure drop is found to increase along with mass flow rate. 


\section{Concluding remarks}

Although the kinetic theory approach provides a rigorous solution to the particulate stresses, many issues still need to be addressed. For example, the spatial homogeneity assumption may be invalid if the inter-particle collisions are not nearly elastic, especially for a dense gas solid flow. The coefficient of restitution, which is assumed constant in current kinetic theory models, depends on impact velocity, which causes great difficulty in tackling spatial inhomogeneity. Moreover, gas turbulence modulation and particle size distribution etc. need to be incorporated in any sophisticated model.

\section{References}

Anderson, T.B. and Jackson, R., 1967, Fluid mechanical description of fluidized beds: comparison with theory and experiment, I\&EC Fund, 6: 527-539.

Brucato, A., Grisafi, F. and Montante, G., 1998, Particle drag coefficients in turbulent fluids, Chem Eng Sci, 53: 3295-3314.

Clift, R., Grace, J.R. and Weber, M.E., 1978, Bubbles, drops, and particles, (Academic Press, London, UK).

Elghobashi, S.E., 1994, On predicting particle-laden turbulent flows, Appl Sci Res, 52: 309329.Goldhirsch, I. and Zanetti, G., 1993, Clustering instability in dissipative gases. Phys Rev Lett, 70: 1619-1622.

Ergun, S., 1952, Fluid flow through packed columns, Chem. Eng. Prog., 48: 89-94.

Gore, R.A. and Crowe, C.T., 1989, The effect of particle size on modulating turbulence intensity, Int J Multiphase Flow, 15: 279-285.

Jenkins, J.T. and Richman, M.W., 1985, Grad's 13-moment system for a dense gas of inelastic spheres, Arch Ration Mech Anal, 87: 3485-3494.

Koch, D.L., 1990, Kinetic theory for a monodisperse gas-solid suspension, Phys Fluids A, 2: 1711-1723. 
Koch, D.L. and Sangani, A.S., 1999, Particle pressure and marginal stability limits for a homogeneous monodisperse gas-fluidized bed: kinetic theory and numerical simulations, J Fluid Mech, 400: 229-263.

Louge, M.Y., Mastorakos, E. and Jenkins, J.K., 1991, The role of particle collisions in pneumatic transport, J Fluid Mech, 231: 345-359.

Lun, C.K.K., Savage, S.B., Jeffrey, D.J. and Chepurniy, N., 1984, Kinetic theory for granular flow: inelastic particles in Couette flow and slightly inelastic particles in a general flowfield, J Fluid Mech 140: 223-256.

Lun, C.K.K. and Savage, S.B., 1987, A simple kinetic theory for granular flow of rough, inelastic, spherical particles, Trans ASME J Appl Mech, 54: 47-53.

Miller, A. and Gidaspow, D., 1992, Dense, vertical gas-solid flow in a pipe, AIChE J, 38: 18011815.

Monceaux, L., Azzi, M., Molodtsof, Y. and Large, J.F., 1986, Overall and local characterisation of flow regimes in a circulating fluidized bed, in Circulating Fluidized Bed Technology I, Basu, P. (eds) (Pergmon Press, Oxford, UK), pp 185-191.

Neri, A. and Gidaspow, D., 2000, Riser hydrodynamics: simulation using kinetic theory, AIChE $J, 46: 52-67$

Nieuwland, J.J., van Sint Annaland, M., Kuipers, J.A.M. and van Swaaij, W.P.M., 1996a, Hydrodynamic modelling of gas/particle flows in riser reactors, AIChE J, 42: 1569-1582.

Nieuwland, J.J., Meijer, R., Kuipers, J.A.M. and van Swaaij, W.P.M., 1996b, Measurements of solid concentration and axial solids velocity in gas-solid two-phase flows, Powder Tech, 87: 127-139.

Ocone, R., Sundaresan, S., and Jackson, R., 1993, Gas-particle flow in a duct of arbitrary inclination with particle-particle interactions, AIChE J, 39: 1261-1271.

Ocone, R., Goodwin, J.A.S. and Delebarre, A., 2000, Flow structures of Geldart A solid in circulating fluidised beds, Chem Eng Res Des, 78: 860-865. 
Peirano, E. and Leckner, B., 1998, Fundamentals of turbulent gas-solid flows applied to circulating fluidized bed combustion, Prog Energy Combust Sci, 24: 259-296.

Rhodes, M.J., Wang, X.S., Cheng, H. and Hirama, T., 1992, Similar profiles of solids flux circulating fluidised-bed risers, Chem Eng Sci 47: 1635-1643.

Sinclair, J.L. and Jackson, R., 1989, Gas-particle flow in a vertical pipe with particle-particle interactions, AIChE J, 35: 1473-1486.

Wen, C.Y. and Yu, Y.H., 1966, A generalized method for predicting the minimum fluidization velocity, AIChE J, 12: 610-612.

Zaichik, L.I., Pershukov, V.A., Kozelev, M.V. and Vinberg, A.A., 1997, Modeling of dynamics, heat transfer, and combustion in two-phase turbulent flows: 1. isothermal flows, Exp Thermal Fluid Sci, 15: 291-310.

Zhang, Y. and Reese, J.M., 2001, Particle-gas turbulence interactions in a kinetic theory approach to granular flows, Int J Multiphase Flow, 27: 1945-1964.

Zhang, Y. and Reese, J.M. 2003, The drag force in two-fluid models of gas-solid flows, Chem Eng Sci, (in press).

\section{Address}

Correspondence concerning this manuscript should be addressed to

Dr Yonghao Zhang, Department of Computational Science and Engineering, CLRC Daresbury Laboratory, Warrington, WA4 4AD, UK.

Email: $\underline{\text { Y.Zhang@dl.ac.uk }}$ 


\section{APPENDIX}

From the work of Peirano and Leckner (1998), the bulk viscosity is

$$
\zeta_{2}=\frac{4}{3} d \varepsilon_{2}^{2} \rho_{2} \chi(1+e) \sqrt{\frac{T}{\pi}}
$$

the collisional viscosity is given by

$$
v_{2}^{c}=\frac{4}{5} \varepsilon_{2} \chi(1+e)\left(v_{2}^{t}+d \sqrt{\frac{T}{\pi}}\right)
$$

and the turbulent viscosity by

$$
v_{2}^{t}=\left(\frac{2}{3} \frac{t_{12}^{t}}{t_{12}^{x}} k_{12}+\left(1+\varepsilon_{2} \chi A\right) T\right) /\left(\frac{2}{t_{12}^{x}}+\frac{B}{t_{2}^{c}}\right),
$$

where $A=\frac{2}{5}(1+e)(3 e-1), B=\frac{1}{5}(1+e)(3-e) ; \quad t_{12}^{t}$ is the interaction time between particle motion and gas fluctuations, which can be evaluated through the characteristic time scale of the eddy, $t_{1}^{t}$; the particle relaxation time is:

$$
t_{12}^{x}=\frac{4 d \rho_{2}}{3 \rho_{1}\left\langle C_{D}\right\rangle\left\langle\left|u_{r}\right|\right\rangle},
$$

and the particle collision time is :

$$
t_{2}^{c}=\frac{d}{24 \varepsilon_{2} \chi} \sqrt{\frac{\pi}{T}} .
$$

The collisional diffusion coefficient is given by

$$
k_{2}^{c}=\varepsilon_{2} \chi(1+e)\left(\frac{6}{5} k_{2}^{t}+\frac{4}{3} d \sqrt{\frac{T}{\pi}}\right),
$$

and the turbulent diffusion coefficient by

$$
k_{2}^{t}=\left[\frac{3 t_{12}^{t}}{5 t_{12}^{x}} k_{12}+\left(1+\varepsilon_{2} \chi C\right) T\right] /\left(\frac{9}{5 t_{12}^{x}}+\frac{D}{t_{2}^{c}}\right),
$$

where $C=3(1+e)^{2}(2 e-1) / 5$ and $D=(1+e)(49-33 e) / 100$. 
Table 1 Summary of the properties of the gas and solid phases used in the calculations, corresponding to the experimental set-up of Nieuwland et al. (1996b).

Gas density, $\rho_{1}\left(\mathrm{~kg} \mathrm{~m}^{-3}\right)$

Gas viscosity, $\mu\left(\mathrm{kg} \mathrm{m}^{-1} \mathrm{~s}^{-1}\right)$

Particle-particle restitution coefficient, $e$

Particle-wall restitution coefficient, $e_{w}$

Particle density, $\rho_{2}\left(\mathrm{kgm}^{-3}\right)$

Mean particle diameter, $d(\mu \mathrm{m})$

Particle diameter distribution $(\mu \mathrm{m})$

Specularity coefficient, $\phi^{*}$

Pipe radius, $R(\mathrm{~mm})$

Measuring height above inlet (m)
1.2

$1.8 \times 10^{-5}$

0.98

0.9 2540

129

$50<d<150$

0.3

27

over 2.5

*Specularity coefficient $\phi$ is a parameter used in the boundary conditions at the wall for the particulate phase and specifies the fraction of the momentum of the incident particle transferred to the wall. 


\section{Figure captions}

Figure 1 Comparison of the present model with the simulation and measurements of Nieuwland et al.(1996a, b), with $G_{s 0}=350 \mathrm{kgm}^{-2} \mathrm{~s}^{-1}, V_{s}=14.4 \mathrm{~ms}^{-1}$, and other parameters as in Table 1. Variation of (a) solid volume fraction, (b) solid axial velocity.

Figure 2 Comparison of the present model with the measurements of Nieuwland et al. (1996b), with $G_{s 0}=400 \mathrm{kgm}^{-2} \mathrm{~s}^{-1}, V_{s}=10 \mathrm{~ms}^{-1}$, and other parameters as in Table 1. Variation of (a) solid volume fraction and (b) solid axial velocity with non-dimensional radius.

Figure 3 Comparison of the present model with the simulation and measurements of Nieuwland et al. (1996a, b), with $G_{s 0}=300 \mathrm{kgm}^{-2} \mathrm{~s}^{-1}, V_{s}=10 \mathrm{~ms}^{-1}$, and other parameters as in Table 1. Variation of (a) solid volume fraction and (b) solid axial velocity with nondimensional radius.

Figure 4 Comparison of the present model with measurements of Nieuwland et al. (1996b), with $G_{s 0}=200 \mathrm{kgm}^{-2} \mathrm{~s}^{-1}, V_{s}=10 \mathrm{~ms}^{-1}$, and other parameters as in Table 1. Variation of (a) solid volume fraction and (b) solid axial velocity with non-dimensional radius.

Figure 5 Similar profiles: the radial variation of reduced solid mass flow rate; $V_{s}=10 \mathrm{~ms}^{-1}$, and other parameters as in Table 1. 

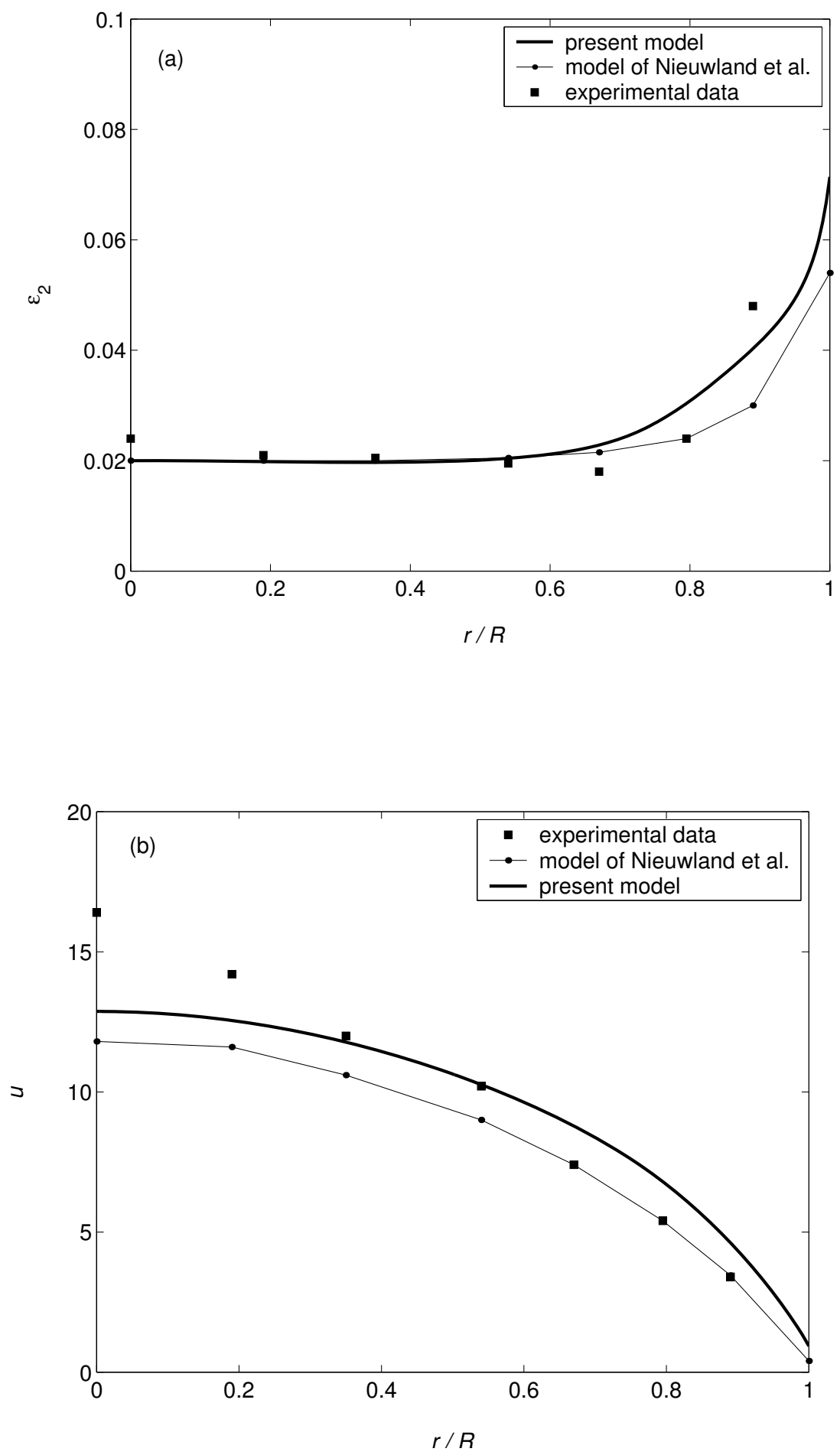

Figure 1 Comparison of the present model with the simulation and measurements of Nieuwland et al. (1996a, b), with $G_{s 0}=350 \mathrm{kgm}^{-2} \mathrm{~s}^{-1}, V_{s}=14.4 \mathrm{~ms}^{-1}$, and other parameters as in Table 1. Variation of (a) solid volume fraction, (b) solid axial velocity. 

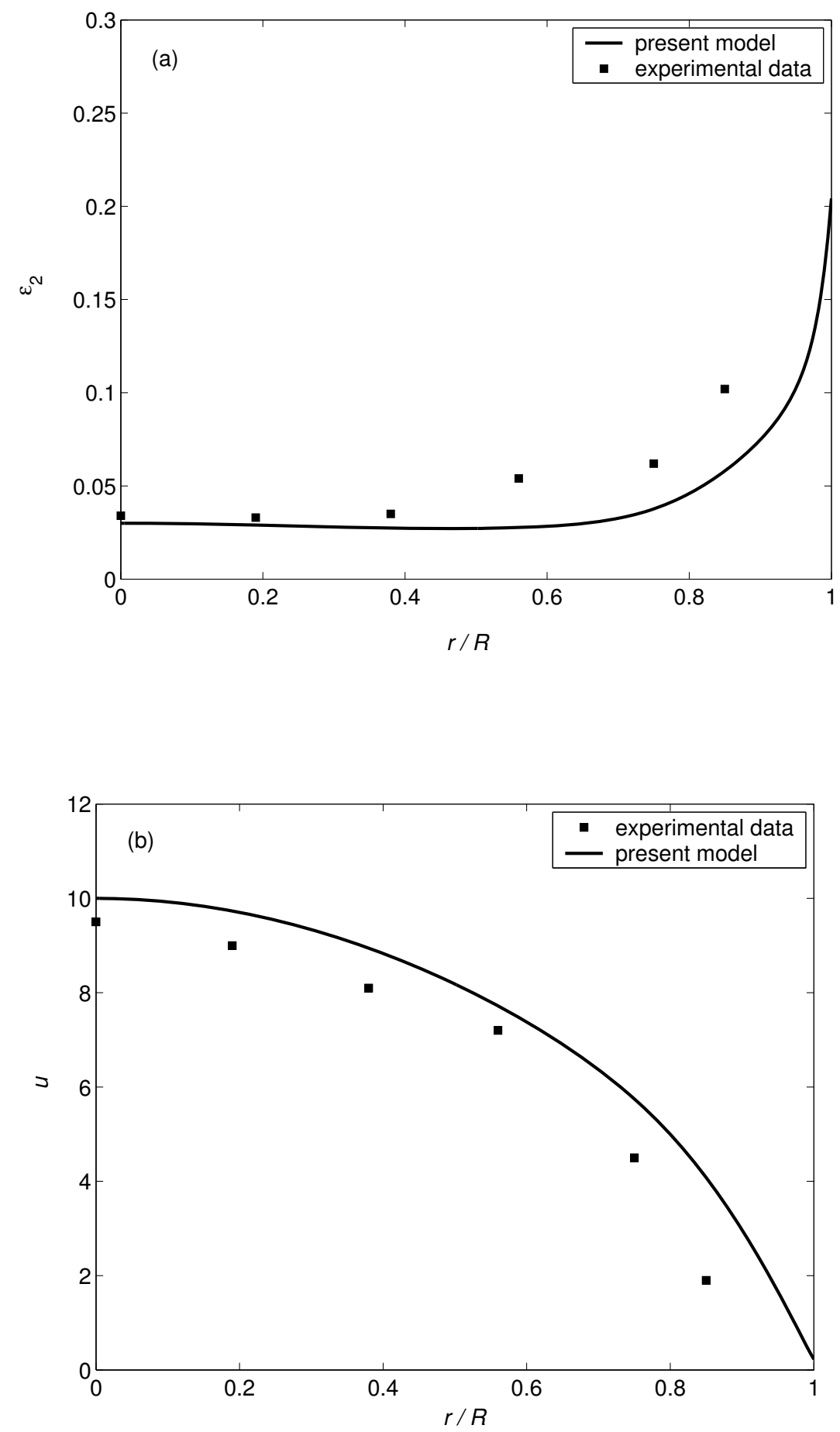

Figure 2 Comparison of the present model with the measurements of Nieuwland et al. (1996b), with $G_{s 0}=400 \mathrm{kgm}^{-2} \mathrm{~s}^{-1}, V_{s}=10 \mathrm{~ms}^{-1}$, and other parameters as in Table 1. Variation of (a) solid volume fraction and (b) solid axial velocity with non-dimensional radius. 

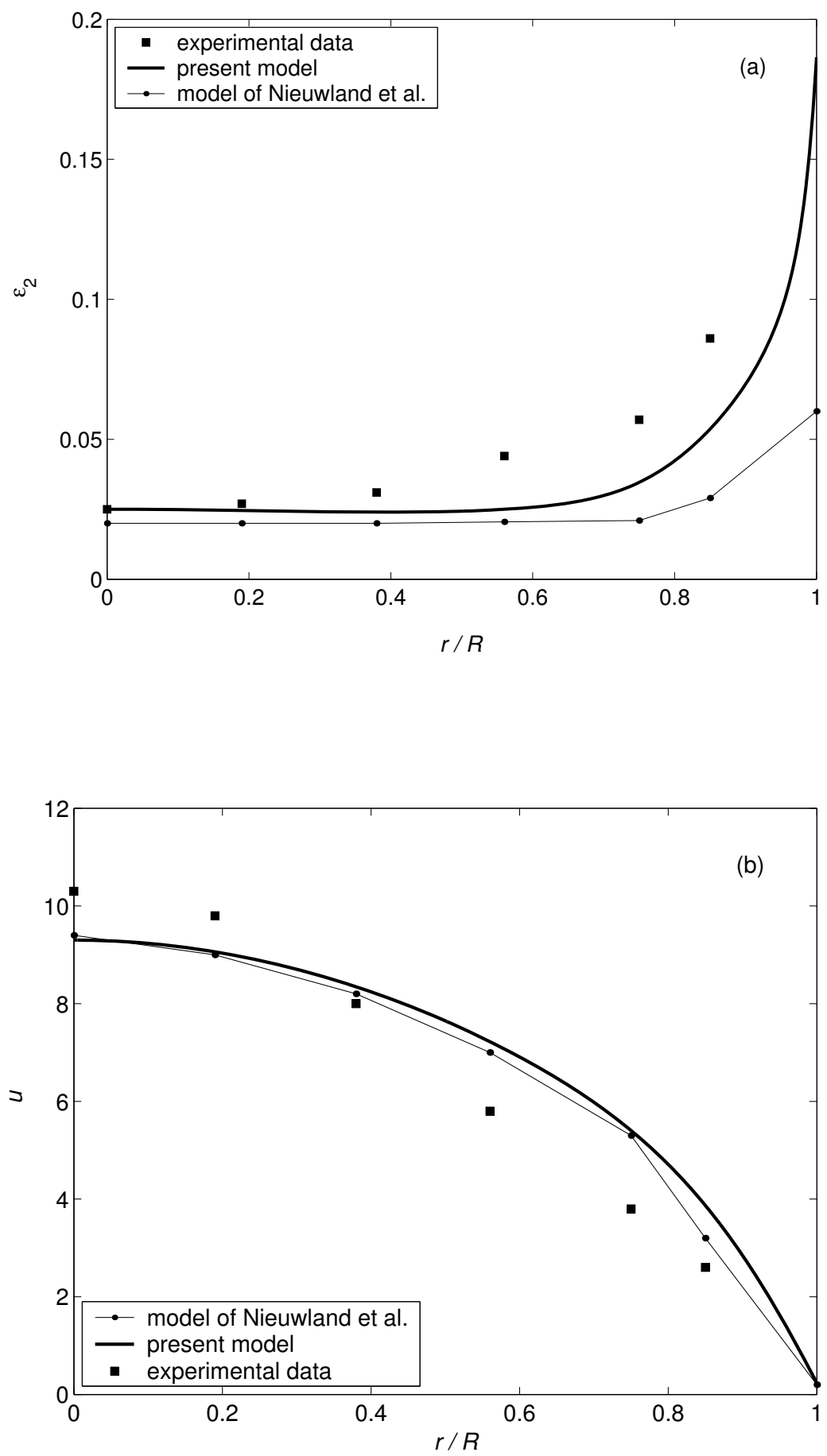

Figure 3 Comparison of the present model with the simulation and measurements of Nieuwland et al. (1996a, b), with $G_{s 0}=300 \mathrm{kgm}^{-2} \mathrm{~s}^{-1}, V_{s}=10 \mathrm{~ms}^{-1}$, and other parameters as in Table 1. Variation of (a) solid volume fraction and (b) solid axial velocity with nondimensional radius. 

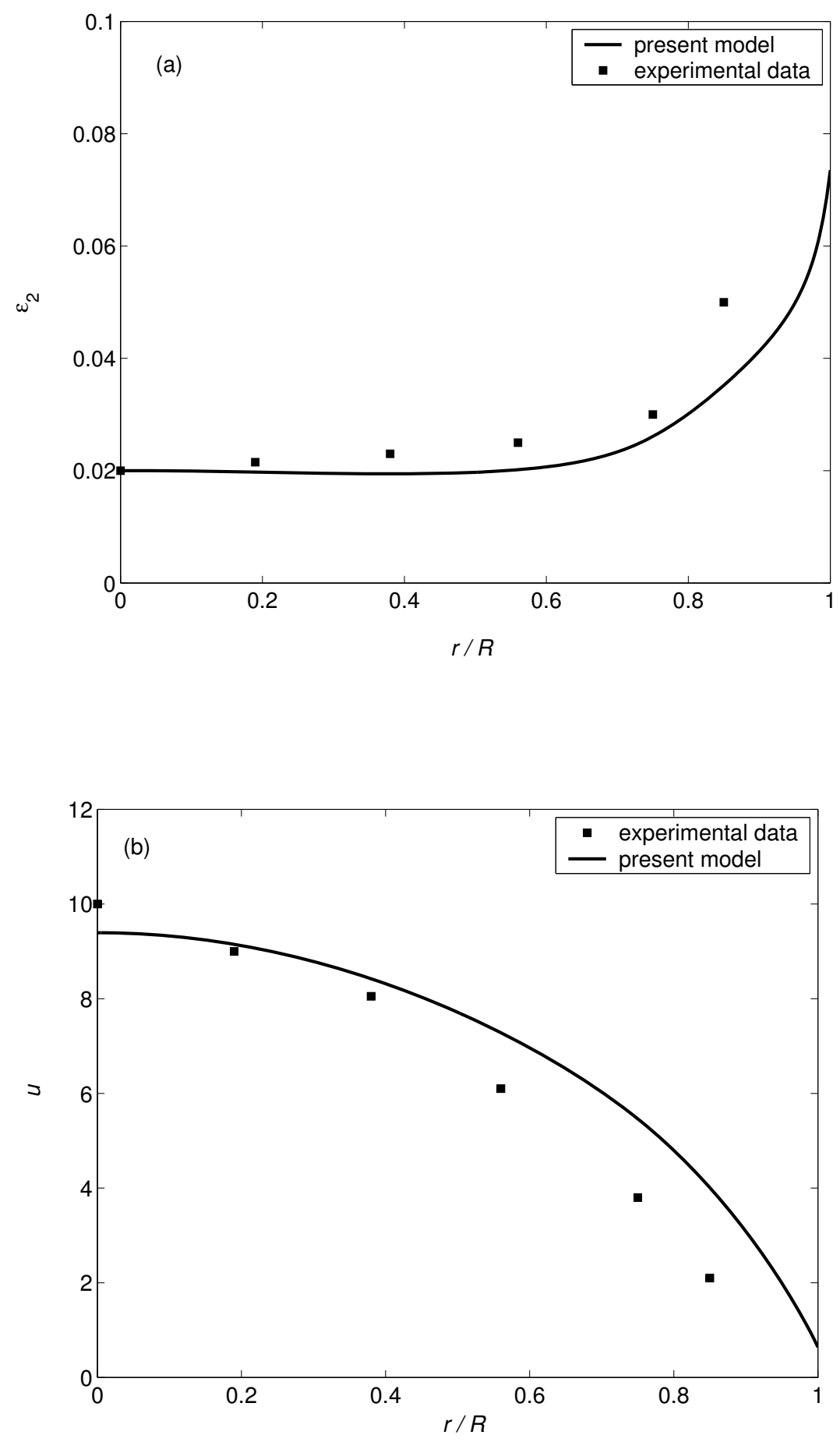

Figure 4 Comparison of the present model with measurements of Nieuwland et al. (1996b), with $G_{s 0}=200 \mathrm{kgm}^{-2} \mathrm{~s}^{-1}, V_{s}=10 \mathrm{~ms}^{-1}$, and other parameters as in Table 1. Variation of (a) solid volume fraction and (b) solid axial velocity with non-dimensional radius. 


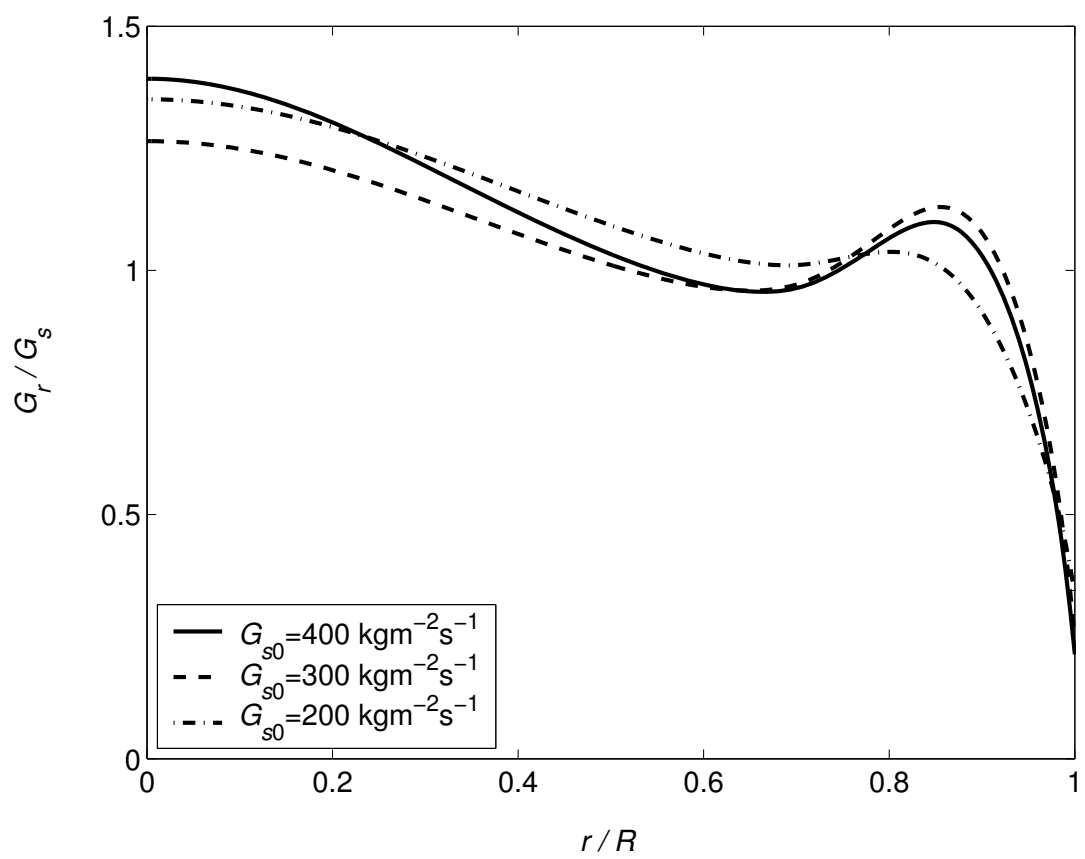

Figure 5 Similar profiles: the radial variation of reduced solid mass flow rate; $V_{s}=10 \mathrm{~ms}^{-1}$, and other parameters as in Table 1. 